\title{
Axillary Masses as Clinical Manifestations of Male Sweat Gland Carcinoma Associated with Extramammary Paget's Disease and Accessory Breast Carcinoma: Two Cases Report and Literature Review
}

\section{Jing Wu}

Chongqing University Cancer Hospital

\section{Hongdan Chen}

Chongqing General Hospital

Juanhui Dong

Chongqing University Cancer Hospital

\section{Yong Cao}

Chongqing University Hopital

Wei Li

Chongqing University Hospital

Fan Zhang

Chongqing General Hospital

Xiaohua Zeng ( $\sim$ zengxiaohua000017@163.com )

Chongqing University Cancer Hospital https://orcid.org/0000-0002-6758-2856

\section{Research Article}

Keywords: Axillary mass, Sweat gland carcinoma, Accessory breast carcinoma, Extramammary Paget's disease

Posted Date: October 21st, 2021

DOl: https://doi.org/10.21203/rs.3.rs-960312/v1

License: (a) This work is licensed under a Creative Commons Attribution 4.0 International License. Read Full License

Version of Record: A version of this preprint was published at World Journal of Surgical Oncology on April 4th, 2022. See the published version at https://doi.org/10.1186/s12957-022-02570-w. 


\section{Abstract}

\section{Background}

Accessory breast carcinoma and sweat gland carcinoma associated with extramammary Paget's disease of the axilla in male are rare cases. In clinical diagnosis and treatment, it is necessary to determine the disease carefully and make a reasonable treatment strategy according to the patient's own situation.

\section{Case presentation}

We reported two male cases of special tumor with axillary mass as initial clinical symptom, one was diagnosed as accessory breast cancer and the other was diagnosed as sweat gland cancer associated with extramammary Paget's disease. We did personalized treatment for the two diseases, hoping to provide a reference for the diagnosis and management of diseases with axillary nodules as the initial symptoms.

\section{Conclusions}

The reports of these two cases have the potential to provide reference and corresponding thinking for clinical differentiation of axillary lymphadenopathy caused by different causes and subsequent treatment. These two cases may further enrich the database of rare cases and provide some ideas for the treatment of axillary lymphadenopathy caused by different causes.

\section{Background}

Accessory breast carcinoma $(A B C)$ occurs anywhere along the milk line, with the axilla being the most commonly involved site, followed by the inframammary area[1]. The incidence of $A B C$ is around $0.2-$ $0.6 \%$ and that of male $A B C$ is more insidious[2]. Sweat gland carcinoma (SGC) is a rare low-grade malignant skin adnexal tumor[3,4]. Clinically, it mostly occurs in the head and neck skin, followed by axillary, chest wall, scrotum and perianal areas with asymptomatic nodular growth. SGC is prone to local recurrence and distant metastasis, and the clinical prognosis is poor[5]. Studies have shown that the incidence rate of SGC is about $0.05 \%$, which accounts for about $2.2 \% \sim 8.4 \%$ of skin malignant tumors. The age of onset is 40-60 years old, and women are more common than men[6, 7]. Extramammary Paget's disease (EMPD) is a rare kind of intraepidermal adenocarcinoma involving Paget cells. It occurs frequently in areas rich in sweat glands and eccrine glands, such as vulva, genitalia and perianal region, and a few can also occur in armpit[8]. Sweat gland carcinoma with EMPD in the axilla is rarer.

In this article, we reported a case of male ABC and a case of male SGC associated with EMPD. The clinical features and the treatment process of these two patients were very similar. They both started with axillary mass and then underwent local mass resection in a hospital near their home. In the absence of imaging evidence, the final diagnosis mainly depended on pathology, for example, the shape and size of cancer cells and immunohistochemical characteristics. Through these two cases, we hope to arouse 
medical practitioner' attention to these rare diseases and provide some evidence for the diagnosis of these two types of diseases.

\section{Case Presentation}

\section{Case 1}

An 83-year-old male patient found a mass in his right axilla 1 year ago without redness, swelling, and ache. Until the tumor gradually increased to about $3 \mathrm{~cm}$ in size, the patient went to the nearest hospital and underwent local tumor resection on October 26,2020. Then he came to our hospital for further treatment. Imaging examinations such as CT, MRI and PET/CT showed no abnormal enhancement or mass in the breast, no enlarged lymph nodes in the right armpit, and no distant metastasis (Fig. 1). Postoperative pathology showed that there was a nodule with skin, $3 \mathrm{~cm}$ in diameter. Histologically, poorly differentiated adenocarcinoma was found in subcutaneous fibrous tissue. The immunohistochemical staining of the infiltrative tumor cell showed positive immunohistochemical staining for anti-gross cystic disease fluid protein-15 (GCDFP-15), GATA-3, cytokeratin (CK), CK7, EMA, and negative for estrogen receptor (ER), progesterone receptor (PR), prostate specific antigen (PSA), CK20, CDX2, p504S, p63, P40, TTF-1, NapsinA, PLAP, Syn, CgA. The expression of HER2 was 2+ by immunohistochemistry and fluorescence in situ hybridization (FISH) showed no amplification, and Ki67 was $20 \%$ positive (Fig. 2). After combining the imaging examination, clinical physical examination and immunohistochemical index, the patient was finally diagnosed as accessory breast cancer.

Considering that the patient was old and had coronary heart disease and serious arrhythmia in the evaluation of cardiopulmonary function, no further lymphadenectomy and chemotherapy were performed. Finally, the patient was treated with intensity modulated radiation therapy (IMRT) with 6MV Xray. The radiotherapy area included the right operation area, the right axillary lymph node drainage area and the right accessory breast area. The radiation dose $60 \mathrm{~Gy} / 30 \mathrm{~F}$ in total. After more than 6 months of follow-up, the patient is healthy and free of symptoms.

\section{Case 2}

A 66-year-old male patient was referred to the nearest clinic for further treatment because he accidentally found a $1.5 \mathrm{~cm}$ mass in the left armpit, bulging and red in appearance, without pain and ulceration. After routine examination, left axillary tumor resection was performed on June 20, 2020 in the nearby hospital. When he came to our hospital, our imaging examinations demonstrated that there was no clear mass in bilateral mammary glands, no obvious enlarged lymph nodes in bilateral armpits, and no metastasis of other organs (Fig. 3). Histologically, the main component of this malignant tumor was adenocarcinoma cell, which tends to be sweat gland origin, co-existing with Paget's cells. Strong positive immunostaining of GCDFP-15, GATA-3, CK, E-cadherin, P120-tcn and partial positive immunostaining of CK5/6, CK20, P53 were observed in sweat gland carcinoma and Paget's cells, whereas ER, PR, P53, Calponin, MelanA, S-100, HMB-45, MUC2 did not stain. The expression of HER2 was 2+ by immunohistochemistry and fluorescence in situ hybridization (FISH) showed amplification, and Ki67 was $20 \%$ positive. In addition, 
androgen receptor (AR) was $40 \%$ positive (Fig. 4). Based on these results, the patient was evaluated for evidence of sweat gland cancer associated with extramammary Paget's disease.

Thirty days after local resection, the patient underwent extended resection of left axillary tumor and left axillary lymph node dissection. The pathology after operation displayed a small number of atypical cells under the mucosa of the left axillary tumor resection tissue, and no metastasis was found in the axillary lymph nodes (0/14). From September 4, 2020 to November 13, 2020, four cycles of TC (docetaxel and cyclophosphamide) regimen were used as adjuvant chemotherapy. So far, the patients were followed up in good health, no recurrence and metastasis.

\section{Discussion And Conclusions}

To our knowledge, $A B C$ in males is extremely rare and only a few cases reported at present[9-14]. Accessory breast tissue is a kind of abnormal breast tissue, and its development and pathology are similar to normal breast tissue. Usually, patients especially male patients can not aware of the existence of accessory mamma, unless it develops into inflammation, benign mass, malignant tumor. Primary accessory breast cancer in axillary accounts for $60-70 \%$ of ectopic breast tumor[15-17]. The most common pathological type is invasive ductal carcinoma, just like normal breast cancer[18]. As ABC is rare, early diagnosis is difficult, often resulting in clinical missed diagnosis and misdiagnosis. The differential diagnosis between breast cancer and $A B C$ mainly depends on anatomical location, imaging examination and pathological diagnosis. In addition, other diseases such as lymph node metastasis, sweat gland cancer and lymph node tumor should be excluded.

The clinical diagnosis of $A B C$ depends on clinical physical examination and imaging examination, such as ultrasound, CT and MRI. Although some studies suggest that ultrasound and MRI can improve the detection rate of $A B C[19,20]$, pathology is still the gold standard of diagnosis. In our case, the tumor has been removed in an outside hospital, so the final diagnosis mainly depends on pathology. In our case, we made a diagnosis of accessory breast carcinoma of the axilla because there was no evidence of other organ cancer especially the mammary gland. Most of the tumors were invasive carcinoma, and there were also a few intraductal components which was the important evidence for the diagnosis. The possibility of metastasis from other sites such as gastrointestinal and pulmonary origin also was excluded by different combination of immunohistochemistry.

Due to the rarity of $A B C$ and the lack of large sample research results, most scholars agree that the treatment of $A B C$ follows the breast cancer guidelines. Some scholars believe that if there is regional lymph node infiltration, radical mastectomy is feasible[21], but Evans et al. [22]found that radical mastectomy or modified radical mastectomy has no obvious advantage in prognosis compared with local resection plus axillary dissection or radiotherapy. Cogwell et al.[23] also pointed out that ipsilateral mastectomy had no significant effect on the prognosis of patients with ectopic breast cancer. Therefore, local resection of negative margin plus axillary lymph node dissection and subsequent radiotherapy, chemotherapy and endocrine therapy are appropriate treatment options. The ABC patient in our case was 
an elderly patient with many underlying diseases, so after comprehensive consideration, we carried out radiotherapy and gave up further lymph node dissection and chemotherapy. Because the patient is triple negative, there is no need for endocrine therapy and targeted therapy.

SGC is a confusing area within dermatopathology with first reported by French pathologist V. Cornil in 1865[24]. Due to a large number of rare entities, a multiplicity of names to designate the same neoplasms and consequent lack of consensus regarding their classification and nomenclature[25] SGC have traditionally subdivided into four broad groups: eccrine, apocrine, mixed origin (eccrine and apocrine) and other un-classifiable sweat gland tumors[4]. SGC is a heterogeneous tumor with different biological behaviors[26]. It generally presents asymptomatic nodular growth, has local invasiveness, and shows high recurrence rate. Immunohistochemistry and molecular genetics play a corresponding role in the diagnosis of sweat gland carcinoma after excluding visceral primary adenocarcinoma and skin metastasis[27]. EMPD is characterized by the invasion of epidermis by Paget cells. The exact pathogenesis of EMPD is not completely clear. The current evidence shows that EMPD is heterogeneous and contains at least two different forms of unique pathogenesis. The primary or cutaneous EMPD forms appear to originate from the skin (epidermis or the underlying apocrine glands) and the secondary form of EMPD is associated with potential (distant) adenocarcinoma[28]. Its immunohistochemical characteristics are usually positive staining for CK7, CEA, EMA, and GCDFP-15[29-31]. SGC co-exists with EMPD are extremely rare. Morgan et al. reported one case of EMPD in axilla accompanied by underlying apocrine carcinoma and they also pointed that $45.5 \%$ (5/11) of previously reported axillary EMPDs were associated with an underlying carcinoma[32]. Chiu et al. reported 1 case of unilateral axillary EMPD having underlying adnexal carcinoma[33]. Jung et al also demonstrated a case of apocrine carcinoma of the axilla associated with EMPD[34]. After we carefully excluded the lesions in other parts or organs, the final diagnosis of our case mainly depended on pathology, especially a large number of adenocarcinoma cells could be seen on HE staining section, and there were also obvious Paget cells on it. Combined with the immunohistochemical characteristics, it is finally considered as SGC coexisted with EMPD.

SGC is easy to recur after operation, so the first surgical resection and lymph node dissection are the key to the treatment of the disease. Bogner et al. reported sentinel lymph node biopsy in 5 patients with sweat gland carcinoma before operation, in which 2 patients had no lymph node metastasis without further surgical treatment. Three patients had lymph node metastasis and then underwent lymph node dissection gradually. No local recurrence or distant metastasis was found in the follow-up of all cases, which demonstrated that sentinel lymph node biopsy should be performed before operation to confirm whether there was metastasis and whether needed further extended resection or regional lymph node dissection[35]. The treatment of EMPD depends on adequate surgical excision that should be sufficiently wide in surface and depth so as to eradicate the entire tumor mass. Although the margin of $1 \mathrm{~cm}$ is enough, $2 \mathrm{~cm}$ is often recommended as a safe margin because the margin of this type of disease is not very clear[36]. The low recurrence rate can be ensured by the negative margin on frozen sections and Mohs graphic surgery[37]. Combined with the characteristics of the above two diseases, our patient finally underwent extended resection and lymphadenectomy. Besides, we also gave the patient systemic chemotherapy (docetaxel and cyclophosphamide) after surgery. Because of his HER2 gene amplification, 
we suggested that he could give anti HER2 targeted therapy such as trastuzumab, but finally the patient refused because of economic reasons.

In general, the incidence of these two diseases reported by us is very low, especially when both of our cases are male. The two patients had the same onset of axillary lymph node mass, but the final diagnosis was different. As the two patients had tumor resection in other hospitals, the information about the initial tumor is lacking, and the later diagnosis mainly depends on pathology.

\section{Abbreviations}

ABC: Accessory breast carcinoma

SGC: Sweat gland carcinoma

EMPD: Extramammary Paget's disease

\section{Declarations}

\section{Ethics approval and consent to participate}

The information of patients was obtained with the informed consent of all participants. We already have received informed consent for publishing clinical data about patients.

\section{Consent for publication}

The individual person's data contained in the manuscript has been obtained consent for publication by patients.

\section{Availability of data and materials}

All data generated or analyzed during this study are included in this published article.

\section{Competing interests}

The all authors declare that we have no known competing financial interests or personal relationships that could have appeared to influence the work reported in this paper.

\section{Funding}

This work was supported by the Natural Science Foundation of Chongqing, China (grant numbers. cstc2020jcyj-msxmX0485 and cstc2020jcyj-msxmX0668).

\section{Authors' contributions}


X.H.Z. and F.Z. conceived and designed the manuscript; J.W. and H.D.C. screened cases and collected the data; W.L provided pathological pictures; J.H.D and Y.C. analyzed the data; J.W and H.D.C. wrote and revised the manuscript.

\section{Acknowledgements}

None

\section{References}

1. Teke Z, Kabay B, Akbulut M, Erdem E. Primary infiltrating ductal carcinoma arising in aberrant breast tissue of the axilla: a rare entity. Report of a case. Tumori. 2008;94(4):577-83.

2. Francone E, Nathan MJ, Murelli F, Bruno MS, Traverso E, Friedman D. Ectopic breast cancer: case report and review of the literature. Aesthetic Plast Surg. 2013;37(4):746-9.

3. Grieco M, Simonacci F, Grignaffini E, Ricci R, Raposio E: Eccrine porocarcinoma: case report and review of the literature. Giornale italiano di dermatologia e venereologia: organo ufficiale, Societa italiana di dermatologia e sifilografia 2020, 155(4):500-504.

4. Kaseb H, Babiker HM. Eccrine Carcinoma. In: StatPearls. Treasure Island (FL): StatPearls Publishing. Copyright (c) 2021, StatPearls Publishing LLC.; 2021.

5. Brenn T. Do not break a sweat: avoiding pitfalls in the diagnosis of sweat gland tumors. Modern pathology: an official journal of the United States Canadian Academy of Pathology Inc. 2020;33(Suppl 1):25-41.

6. Nair PA, Rathod KM, Chaudhary AH, Pilani AP. Sweat gland adenocarcinoma of scalp. International journal of trichology. 2013;5(4):208-10.

7. Nizawa T, Oshitari T, Kimoto R, Kajita F, Yotsukura J, Asanagi K, Baba T, Takahashi Y, Oide T, Kiyokawa T, et al. Early-stage mucinous sweat gland adenocarcinoma of eyelid. Clinical ophthalmology (Auckland NZ). 2011;5:687-9.

8. Lam C, Funaro D. Extramammary Paget's disease: Summary of current knowledge. Dermatologic clinics. 2010;28(4):807-26.

9. Bi L, Li J, Shi Z, Zhu Z, Lu Z. Male accessory breast cancer successfully treated with endocrine therapy: A case report. Oncology letters. 2015;10(4):2495-8.

10. Bi M, Li D, Su Y, Sun P, Gao Y. Male axillary accessory breast cancer: A case report. Medicine. 2020;99(11):e19506.

11. Lin Y, Wang Y. Case report of a male primary breast carcinoma of axillary accessory mammary gland. Clin Breast Cancer. 2012;12(2):142-4.

12. Yoshida Y, Sakakibara A, Watanabe T, Noto K, Sakita K, Sakai Y, Amemiya T, Akiyama M. Extraordinarily large protruding accessory breast cancer in a man. J Am Acad Dermatol. 2012;67(5):e230-1. 
13. Zhong GB, Ye XQ, Liu JL, Xiao SZ, Huang QH, Wei W: Male accessory breast cancer on the abdominal wall: a case report and literature review. OncoTargets and therapy 2018, 11:6625-6631.

14. Takahashi E, Terata K, Nanjo H, Ishiyama K, Hiroshima Y, Yamaguchi A, Yatsuyanagi M, Kudo C, Wakita A, Takashima S, et al: A male with primary accessory breast carcinoma in an axilla is strongly suspected of having hereditary breast cancer. International cancer conference journal 2021, 10(2):107-111.

15. Amsler E, Sigal-Zafrani B, Marinho E, Aractingi S. [Ectopic breast cancer of the axilla]. Annales de dermatologie et de venereologie. 2002;129(12):1389-91.

16. Marshall MB, Moynihan JJ, Frost A, Evans SR. Ectopic breast cancer: case report and literature review. Surg Oncol. 1994;3(5):295-304.

17. Youn HJ, Jung SH. Accessory Breast Carcinoma. Breast care (Basel Switzerland). 2009;4(2):104-6.

18. Yerra L, Karnad AB, Votaw ML. Primary breast cancer in aberrant breast tissue in the axilla. Southern medical journal. 1997;90(6):661-2.

19. Shi DM, Yong-Tao LU, Zhe XU. De-Xiang YUJPiMB: Value of Ultrasonography in Diagnosis of Breast Cancer. 2011.

20. Capobianco G, Spaliviero B, Dessole S, Rocca PC, Cherchi PL, Ambrosini G, Meloni F, Meloni GB. Lymph node axillary metastasis from occult contralateral infiltrating lobular carcinoma arising in accessory breast: MRI diagnosis. The breast journal. 2007;13(3):305-7.

21. Tjalma WA, Senten LL. The management of ectopic breast cancer--case report. Eur J Gynaecol Oncol. 2006;27(4):414-6.

22. Markopoulos C, Kouskos E, Kontzoglou K, Gogas G. Gogas JJEjogo: Breast cancer in ectopic breast tissue. 2001, 22(2):157-159.

23. Cogswell HD, Czerny EW. Carcinoma of aberrant breast of the axilla. Am Surg. 1961;27:388-90.

24. Gates O, Warren S, Warvi WNJAJOP. Tumors of sweat glands. 1943, 19(4):591-631.

25. Ahn CS, Sangüeza OP. Malignant Sweat Gland Tumors. Hematol Oncol Clin N Am. 2019;33(1):5371.

26. Larson K, Babiker HM, Kovoor A, Liau J, Eldersveld J, Elquza E. Oral Capecitabine Achieves Response in Metastatic Eccrine Carcinoma. Case reports in oncological medicine 2018, 2018:7127048.

27. van der Horst MPJ, Brenn T. Update on Malignant Sweat Gland Tumors. Surgical pathology clinics. 2017;10(2):383-97.

28. Kanitakis J. Mammary and extramammary Paget's disease. Journal of the European Academy of Dermatology Venereology: JEADV. 2007;21(5):581-90.

29. Liegl B, Leibl S, Gogg-Kamerer M, Tessaro B, Horn LC, Moinfar F. Mammary and extramammary Paget's disease: an immunohistochemical study of 83 cases. Histopathology. 2007;50(4):439-47.

30. Shepherd V, Davidson EJ, Davies-Humphreys J. Extramammary Paget's disease. BJOG: an international journal of obstetrics gynaecology. 2005;112(3):273-9. 
31. Yang WJ, Kim DS, Im YJ, Cho KS, Rha KH, Cho NH, Choi YD. Extramammary Paget's disease of penis and scrotum. Urology. 2005;65(5):972-5.

32. Morgan JM, Carmichael AJ, Ritchie C. Extramammary Paget's disease of the axilla with an underlying apocrine carcinoma. Acta dermato-venereologica. 1996;76(2):173-4.

33. Chiu CS, Yang $\mathrm{CH}$, Chen $\mathrm{CH}$. Extramammary Paget's disease of the unilateral axilla: a review of seven cases in a 20-year experience. International journal of dermatology. 2011;50(2):157-60.

34. Jung HR, Kwon SY, Son D. Apocrine Carcinoma of the Axilla Associated with Extramammary Paget's Disease: A Case Report and Review of the Literature. Journal of pathology translational medicine. 2015;49(6):535-7.

35. Bogner PN, Fullen DR, Lowe L, Paulino A, Biermann JS, Sondak VK, Su LD. Lymphatic mapping and sentinel lymph node biopsy in the detection of early metastasis from sweat gland carcinoma. Cancer. 2003;97(9):2285-9.

36. Murata Y, Kumano K. Extramammary Paget's disease of the genitalia with clinically clear margins can be adequately resected with $1 \mathrm{~cm}$ margin. European journal of dermatology: EJD. 2005;15(3):168-70.

37. Hendi A, Brodland DG, Zitelli JA. Extramammary Paget's disease: surgical treatment with Mohs micrographic surgery. J Am Acad Dermatol. 2004;51(5):767-73.

\section{Figures}
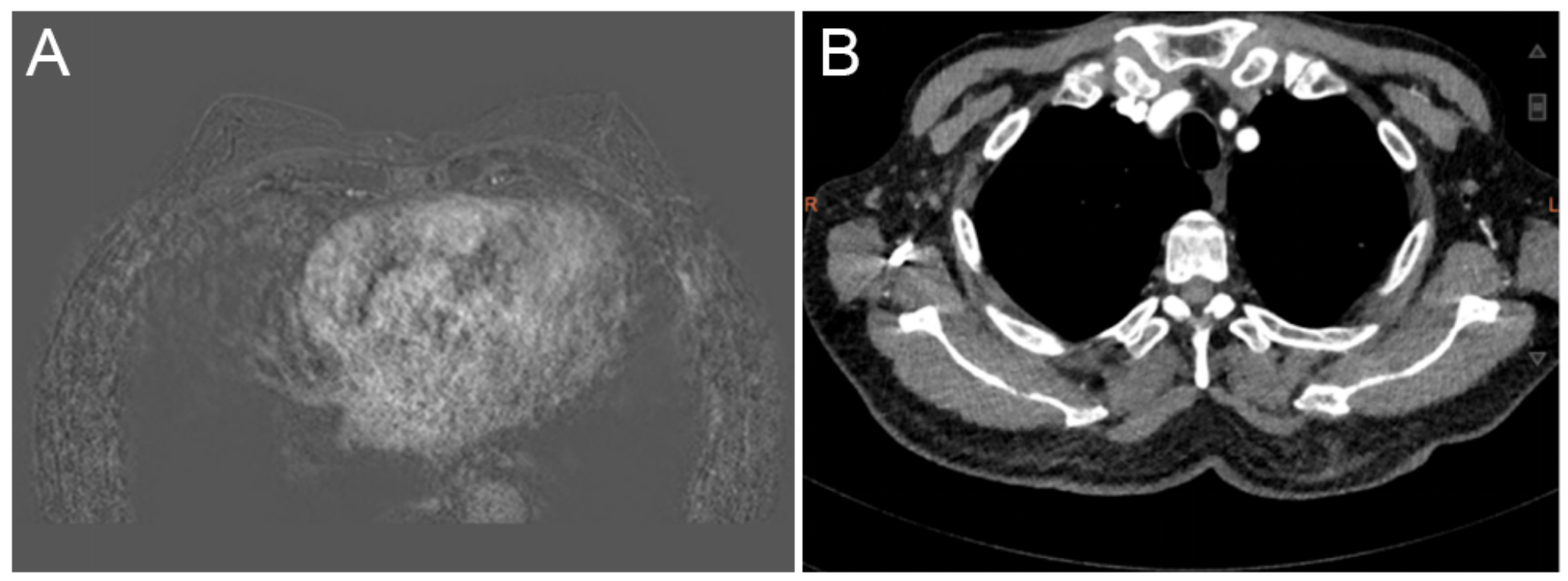

\section{Figure 1}

Imaging examination findings of patient with accessory breast cancer A: Breast MRI showed no abnormal mass. B: CT showed no enlarged lymph nodes in the right armpit. 


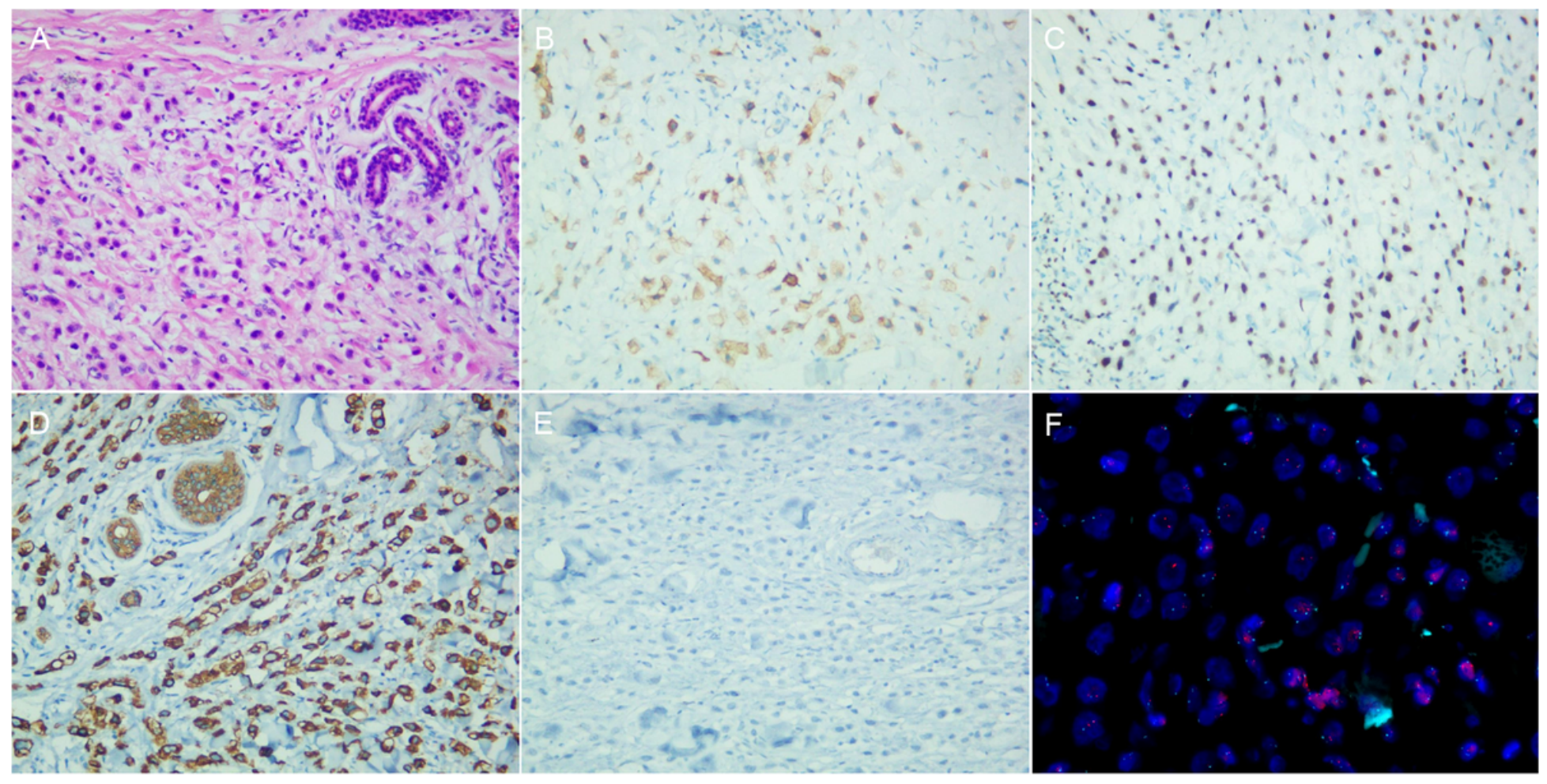

\section{Figure 2}

Immunohistochemical staining results of patient with accessory breast cancer A: Photomicrograph showed poorly differentiated adenocarcinoma (hematoxilin-eosin stain $\times 20$ ). B: GCDFP-15 was positive. C: GATA-3 was positive. D: CK7 was positive. E: CK20 was negative. F: FISH showed no HER2 amplification.
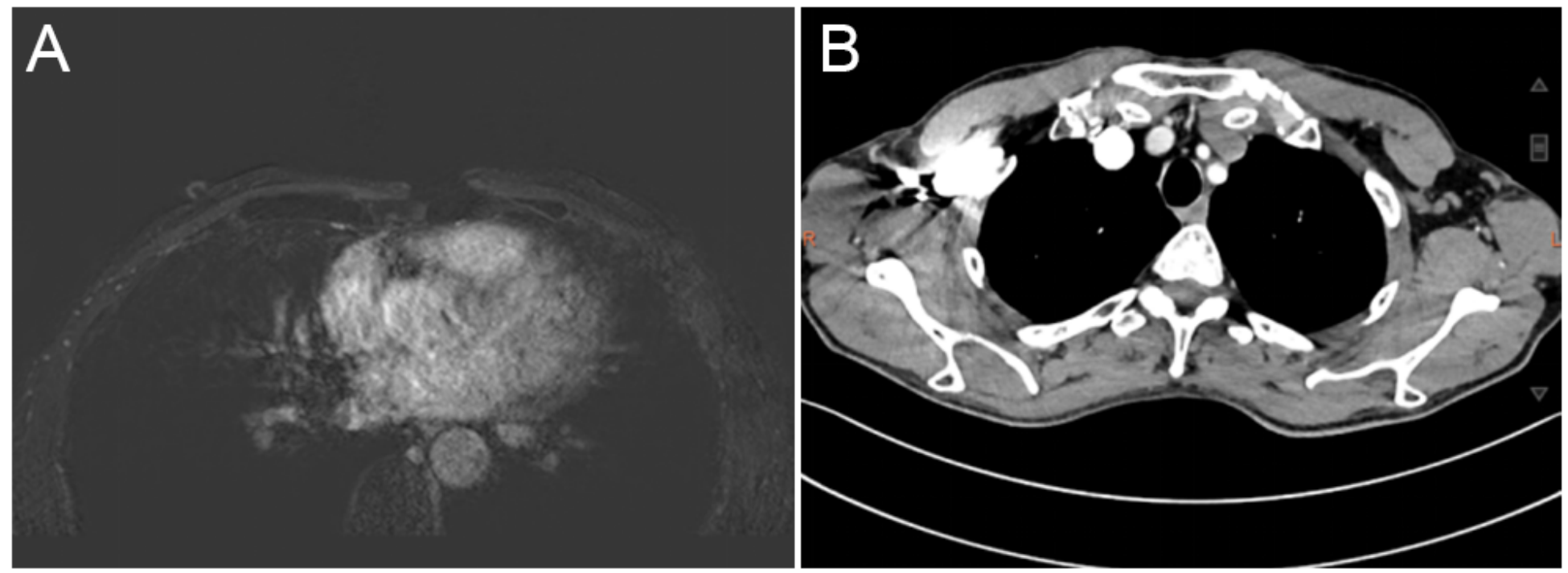

\section{Figure 3}

Imaging examination findings of patient with sweat gland cancer A: Breast MRI showed no abnormal mass. B: CT showed no enlarged lymph nodes in the left armpit. 


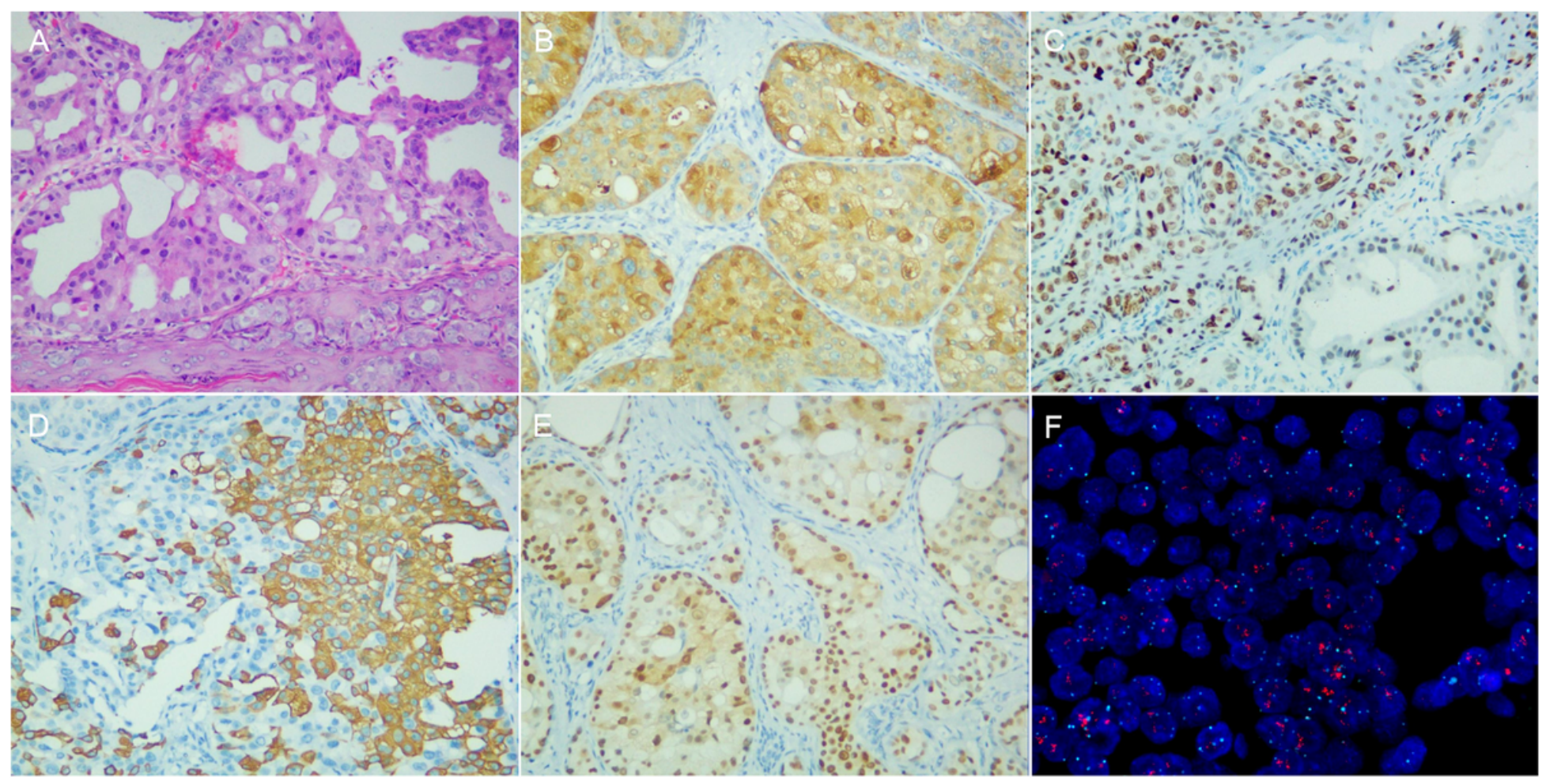

Figure 4

Immunohistochemical staining results of patient with sweat gland cancer A: Histologically showed adenocarcinoma cells co-existing with Paget cells (hematoxilin-eosin stain $\times 20$ ). B: GCDFP-15 was positive. C: GATA-3 was positive. D: CK20 was partial positive. E: AR was positive. F: FISH showed HER2 amplification. 